\title{
Uniqueness of Bogomol'nyi equations and Born-Infeld like supersymmetric theories
}

\author{
H.R. Christiansen ${ }^{a}$, C. Núñez ${ }^{b}$ and F.A. Schaposnik ${ }^{b *}$ \\ ${ }^{a}$ Centro Brasileiro de Pesquisas Físicas, CBPF-DCP \\ Rua Dr. Xavier Sigaud 150, 22290-180 Rio de Janeiro, Brazil \\ ${ }^{b}$ Departamento de Física, Universidad Nacional de La Plata \\ C.C. 67, 1900 La Plata, Argentina
}

June 10, 2021

\begin{abstract}
We discuss Bogomol'nyi equations for general gauge theories (depending on the two Maxwell invariants $F^{\mu \nu} F_{\mu \nu}$ and $\tilde{F}^{\mu \nu} F_{\mu \nu}$ ) coupled to Higgs scalars. By analysing their supersymmetric extension, we explicitly show why the resulting BPS structure is insensitive to the particular form of the gauge Lagrangian: Maxwell, Born-Infeld or more complicated non-polynomial Lagrangians all satisfy the same Bogomol'nyi equations and bounds which are dictated by the underlying supersymmetry algebra.
\end{abstract}

\footnotetext{
${ }^{*}$ Investigador CICBA
} 
Supersymmetric extensions of Born-Infeld theories and the corresponding Bogomol'nyi equations play a central rôle in the dynamics of D-branes and for this reason they have recently received a lot of attention [1]- 8]. SUSY Born-Infeld theories were originally studied in [9]-[10] (see also [11]) while for the Bogomol'nyi equations in BornInfeld systems, early constructions were reported in [12-13. More recent analyses of these issues were presented in [7]-[8], [14].

When studied à la Bogomol'nyi, Born-Infeld theories were coupled to Higgs scalars so as to reproduce the ordinary (i.e. Maxwell or Yang-Mills) BPS relations [12]- [13]. From the supersymmetry point of view, the usual BPS equations arise naturally on very general grounds [6]-[8], [14]. As a consequence of these results, the question on whether BPS relations are or not sensitive to the dynamics that one choses for the gauge field is then posed. It is the purpose of this letter to give an answer to this question.

A first quick answer can be drawn by observing that in the SUSY framework, one obtains Bogomol'nyi equations by imposing the vanishing of (half of) the supersymmetry variations of the gaugino and higgsino fields and these variations are formally the same for very different Lagrangians. The dynamics associated with the Lagrangian enters however through the equation of motion for the auxiliary field $D$ (of the gauge field supermultiplet) which appears in the supersymmetric transformation law for the gaugino. It is then through $D$ that the form of the Lagrangian may in principle determine the form of the BPS relations.

What we show in this work is precisely that supersymmetry together with the (algebraic) equation of motion for $D$ make the BPS relations remain unchanged irrespectively of the choice of the gauge field Lagrangian.

We present our analysis by considering an Abelian gauge theory in $d=3$ dimensions, for which the Bogomol'nyi equations are those originally derived in [17-18 for a Maxwell action; our arguments should hold, however, for other models like for example the $S O(3)$ gauge theory and in other dimensionalities of space-time.

Our conventions will be those of reference [14].

We first consider $d=4$ dimensional Minkowski space (with signature $(+,-,-,-)$ ) and then proceed to dimensional reduction to $d=3$. The gauge vector superfield $V$ is written, in the Wess-Zumino gauge,

$$
V=-\theta \sigma^{\mu} \bar{\theta} A_{\mu}+i \theta \theta \bar{\theta} \bar{\lambda}-i \bar{\theta} \bar{\theta} \theta \lambda+\frac{1}{2} \theta \theta \bar{\theta} \bar{\theta} D
$$

Here $A_{\mu}$ is a vector field, $\lambda=\left(\lambda_{\alpha}\right)$ and $\bar{\lambda}=\left(\bar{\lambda}^{\dot{\alpha}}\right)$ are two-component spinors $(\alpha, \dot{\alpha}=$ 
1,2 ) which can be combined to give a four-component Majorana fermion and $D$ is an auxiliary field.

From $V$ a chiral superfield $W_{\alpha}$ can be constructed,

$$
W_{\alpha}(y, \theta, \bar{\theta})=-i \lambda_{\alpha}+\theta_{\alpha} D-\frac{i}{2}\left(\sigma^{\mu} \bar{\sigma}^{\nu} \theta\right)_{\alpha} F_{\mu \nu}+\theta \theta\left(\sigma^{\mu} \partial_{\mu} \bar{\lambda}\right)_{\alpha}
$$

Here $\lambda, \bar{\lambda}, D$ and $F_{\mu \nu}=\partial_{\mu} A_{\nu}-\partial_{\nu} A_{\mu}$ are functions of the variable $y^{\mu}=x^{\mu}+i \theta \sigma^{\mu} \bar{\theta}$ where $x^{\mu}$ is the usual 4-vector position. The SUSY extension of (standard) gauge-invariant (Maxwell, Yang-Mills) theories are precisely constructed from $W$ by considering $W^{2}$ and its hermitian conjugate $\bar{W}^{2}$.

Now, as stressed in [9], another superfield combination enters into play if one wishes to construct general gauge invariant SUSY Lagrangians. In particular, one needs to consider two superfields $X$ and $Y$ defined as

$$
\begin{gathered}
X=\frac{1}{8}\left(D^{\alpha} D_{\alpha} W^{2}+\bar{D}_{\dot{\alpha}} \bar{D}^{\dot{\alpha}} \bar{W}^{2}\right) \\
Y=-\frac{i}{16}\left(D^{\alpha} D_{\alpha} W^{2}-\bar{D}_{\dot{\alpha}} \bar{D}^{\dot{\alpha}} \bar{W}^{2}\right)
\end{gathered}
$$

with covariant derivatives given by

$$
D_{\alpha}=\frac{\partial}{\partial \theta^{\alpha}}+2 i\left(\sigma^{\mu} \bar{\theta}\right)_{\alpha} \frac{\partial}{\partial y^{\mu}}, \quad \bar{D}_{\dot{\alpha}}=-\frac{\partial}{\partial \bar{\theta}^{\dot{\alpha}}}
$$

when acting on functions of $(y, \theta, \bar{\theta})$ and

$$
D_{\alpha}=\frac{\partial}{\partial \theta^{\alpha}}, \quad \bar{D}_{\dot{\alpha}}=-\frac{\partial}{\partial \bar{\theta}^{\dot{\alpha}}}-2 i\left(\theta \sigma^{\mu}\right)_{\dot{\alpha}} \frac{\partial}{\partial y^{\dagger \mu}}
$$

on functions of $\left(y^{\dagger}, \theta, \bar{\theta}\right)$. The only components of these superfields having purely bosonic terms are

$$
\begin{aligned}
\left.X\right|_{0} & =-\left(D^{2}-\frac{1}{2} F^{\mu \nu} F_{\mu \nu}-i \lambda \not \partial \bar{\lambda}-i \bar{\lambda} \bar{\phi} \lambda\right) \\
\left.X\right|_{\theta \bar{\theta}} & =i \theta \sigma^{p} \bar{\theta} \partial_{p}\left(D^{2}-\frac{1}{2} F^{\mu \nu} F_{\mu \nu}-i \lambda \not \partial \bar{\lambda}-i \bar{\lambda} \bar{\phi} \lambda\right) \\
\left.X\right|_{\theta \bar{\theta} \theta \bar{\theta}} & =\frac{1}{4} \theta \bar{\theta} \theta \bar{\theta} \square\left(D^{2}-\frac{1}{2} F^{\mu \nu} F_{\mu \nu}-i \lambda \not \partial \bar{\lambda}-i \bar{\lambda} \bar{\phi} \lambda\right)
\end{aligned}
$$


and

$$
\begin{aligned}
\left.Y\right|_{0} & =\frac{1}{2}\left(\frac{1}{2} F^{\mu \nu} \tilde{F}_{\mu \nu}+\lambda \not \partial \bar{\lambda}-\bar{\lambda} \bar{\partial} \lambda\right) \\
\left.Y\right|_{\theta \bar{\theta}} & =-\frac{i}{2} \theta \sigma^{p} \bar{\theta} \partial_{p}\left(\frac{1}{2} F^{\mu \nu} \tilde{F}_{\mu \nu}+\lambda \not \partial \bar{\lambda}-\bar{\lambda} \bar{\phi} \lambda\right) \\
\left.Y\right|_{\theta \bar{\theta} \theta \bar{\theta}} & =\frac{1}{8} \theta \bar{\theta} \theta \bar{\theta} \square\left(\frac{1}{2} F^{\mu \nu} \tilde{F}_{\mu \nu}+\lambda \not \partial \bar{\lambda}-\bar{\lambda} \bar{\phi} \lambda\right)
\end{aligned}
$$

with $\tilde{F}_{\mu \nu}=(1 / 2) \varepsilon_{\mu \nu \alpha \beta} F^{\alpha \beta}$.

A third superfield combination is necessary for constructing general gauge invariant SUSY Lagrangian. This combination is $W^{2} \bar{W}^{2}$ with its highest component taking the form

$$
\left.W^{2} \bar{W}^{2}\right|_{\theta \theta \bar{\theta} \bar{\theta}}=\theta \theta \bar{\theta} \bar{\theta}\left(\left(D^{2}-\frac{1}{2} F_{\mu \nu} F^{\mu \nu}\right)^{2}+\left(\frac{1}{2} \tilde{F}_{\mu \nu} F^{\mu \nu}\right)^{2}\right)
$$

Remark that again, all the dependence of (9) on the curvature $F_{\mu \nu}$ and the auxiliary field $D$ is through the combination

$$
t=\frac{1}{\beta^{2}}\left(D^{2}-\frac{1}{2} F^{\mu \nu} F_{\mu \nu}\right)
$$

and this fact will have important consequences in our discussion. Here, in order to define a dimensionless variable $t$ we have introduced a parameter $\beta$ with the same dimensions as $F_{\mu \nu}$ (i.e. dimensions of a mass in $d=4$ ). It corresponds to the absolute field in the Born-Infeld theory [15]-16] as will become clear below.

We are ready to write a general $N=1$ supersymmetric Lagrangian endowed with gauge-invariance in terms of $X, Y$ and $W^{2} \bar{W}^{2}$

$$
L^{d=4}=\frac{1}{4 e^{2}} \int\left(W^{2} d^{2} \theta+\bar{W}^{2} d^{2} \bar{\theta}\right)+\frac{1}{e^{2}} \sum_{r, s, t=0}^{\infty} a_{r s t} \int d^{4} \theta\left(W^{2} \bar{W}^{2}\right)^{r} X^{s} Y^{t}
$$

with $e$ the fundamental gauge coupling constant, which has been factorized in both terms for later convenience.

As it happens for the last component of $W^{2} \bar{W}^{2}$, the first term in the r.h.s. of eq.(11]) depends on $F_{\mu \nu}$ and $D$ through the combination (10). Indeed, the last component in $W^{2}\left(\bar{W}^{2}\right)$ contains the term $D^{2}-\frac{1}{2} F^{\mu \nu} F_{\mu \nu}+i F_{\mu \nu} \tilde{F}^{\mu \nu}\left(D^{2}-\frac{1}{2} F^{\mu \nu} F_{\mu \nu}-i F_{\mu \nu} \tilde{F}^{\mu \nu}\right)$ so that the sum of $\theta(\bar{\theta})$ integrals leads to the well-known SUSY extension of the Maxwell theory. The second term accounts for the non-polynomial features of the general bosonic theory to be supersymmetrized. As explained in [9], supersymmetry 
imposes two constraints on coefficients $a_{r s t}$. Their explicit form will not be relevant for our discussion. What one should retain is that expression (11) gives then the most general Lagrangian corresponding to the supersymmetric extension of a general bosonic Lagrangian depending on the two algebraic Maxwell invariants $F^{\mu \nu} F_{\mu \nu}$ and $\tilde{F}^{\mu \nu} F_{\mu \nu}$.

As stated above, our actual interest is focused on a $d=3, N=2$ supersymmetric theory which can be obtained from Lagrangian (11) by dimensional reduction. The standard procedure for dimensional reduction, say in the $x_{3}$ spatial coordinate, implies identifying $A_{3}$ with a scalar field $N$. Now, it can be shown that without including a Chern-Simons term, the bosonic part of the Lagrangian (11) can only yield electrically neutral configurations, so that as long as one looks for self-dual equations associated with (static) vortices, the $A_{0}$ field (as well as the $N$ field) can be put to zero and so we will do from here on (the case $N \neq 0$ can be equally treated without additional complications). So far, without the addition of a Chern-Simons term, no electrically charged vortices exist and then the most general gauge field configurations are pure magnetic Nielsen-Olesen type soliton solutions [19. This implies that no $d=3$ version of the $\tilde{F}_{\mu \nu} F^{\mu \nu}$ functional are available and that we can simply identify the field strength with the magnetic field $B$ by

$$
\frac{1}{2} F_{\mu \nu} F^{\mu \nu}=B^{2}
$$

with

$$
B=\frac{1}{2} \varepsilon_{j k} F^{j k} \quad i, j=1,2
$$

Once the dimensional reduction is carried on, one ends with the $d=3$ version of the SUSY Lagrangian given in eq.(11). As it is well known, supersymmetry can be extended from $N=1$ to $N=2$ in this process.

From what we have seen, the gauge field dependent terms in the bosonic part of this $N=2$ supersymmetric Lagrangian can be compactly written in the form

$$
L_{A}\left[A_{\mu}, D\right]=\frac{1}{e^{2}} \sum_{n=0}^{\infty} c_{n} t^{n}
$$

where $t$ (defined in (10)) now reads

$$
t=\frac{D^{2}-B^{2}}{\beta^{2}}
$$

and $c_{n}$ are some coefficients which can be computed in terms of the $a_{r s t}$ 's. 
Concerning the Higgs field sector, in $d=4$ dimensions the coupling between the scalar Higgs field $\phi$ and the gauge field $A_{\mu}$ arises from the superfield interaction term

$$
L_{A-\phi}^{d=4}=\Phi \exp (V) \Phi^{*}
$$

where $\Phi$ is a chiral scalar superfield containing a Higgs field $\phi$, a higgsino $\psi$ and an auxiliary field $F$. One can easily see that the part of $L_{A-\phi}$ containing the auxiliary field $D$ is [20]

$$
\left.L_{A-\phi}^{d=4}\right|_{D}=\frac{1}{2} D|\phi|^{2}
$$

On the other hand, gauge symmetry breaking can be achieved $\grave{a}$ la Fayet-Iliopulos so that the complete $D$ dependence of the supersymmetric Lagrangian arising from the Higgs coupling to $A_{\mu}$ and $D$ is given by

$$
\left.L_{D}^{d=4}[A, \phi, D] \equiv L_{A-\phi}^{d=4}\right|_{D}+L_{F I}^{d=4}=\frac{1}{2} D\left(|\phi|^{2}-\xi^{2}\right)
$$

where $\xi$ is a real constant. This Lagrangian remains unchanged after dimensional reduction so that we can write the $D$ dependent terms of the $d=3$ bosonic part of the Lagrangian as

$$
L_{D}^{\text {total }}[A, \phi, D]=\frac{1}{e^{2}} \sum_{n=0}^{\infty} c_{n}\left(\frac{1}{\beta^{2}}\left(D^{2}-B^{2}\right)\right)^{n}+\frac{1}{2} D\left(|\phi|^{2}-\xi^{2}\right)
$$

In $d=3$ space-time, dimensions of parameters and fields are $[\beta]=m^{2},[e]=m^{\frac{1}{2}}$, $[\xi]=m^{\frac{1}{2}},\left[A_{\mu}\right]=m,[D]=m^{2}$ and $[\phi]=m^{\frac{1}{2}}$. Then, for dimensional reasons, one can infer that coefficients $c_{n}^{\prime} s$ can be written in the form

$$
c_{n}=\beta^{2} \lambda_{n}
$$

where $\lambda_{n}$ are dimensionless coefficients. It should be noted that the choice of $\lambda_{1}=$ $-1 / 2, \lambda_{n}=0$ for $n \neq 1$ corresponds to the usual value of the Maxwell term while the choice $\lambda_{1}=-1 / 2, \lambda_{2}=1 / 8, \lambda_{3}=1 / 32, \ldots$, gives a Born-Infeld Lagrangian for the gauge field.

We can now obtain the equation of motion for $D$ so as to eliminate the auxiliary field from the physical spectrum

$$
\sum_{n=0}^{\infty} \frac{2 n}{e^{2}} \lambda_{n}\left(\frac{1}{\beta^{2}}\left(D^{2}-B^{2}\right)\right)^{n-1} D+\frac{1}{2}\left(|\phi|^{2}-\xi^{2}\right)=0
$$


One can easily see that the only nontrivial solution to eq.(21) takes the form

$$
\begin{aligned}
D & =-\frac{e^{2}}{4 \lambda_{1}}\left(|\phi|^{2}-\xi^{2}\right) \\
B & = \pm D
\end{aligned}
$$

These two equations can be readily combined into one which is nothing but the wellhonored Bogomol'nyi equation for the magnetic field of the Nielsen-Olesen vortices

$$
B=\mp \frac{e^{2}}{4 \lambda_{1}}\left(|\phi|^{2}-\xi^{2}\right)
$$

This shows that the Bogomol'nyi gauge field equation for vortex configurations is independent of the particular form of the gauge field Lagrangian one chooses since we have proven formula (23) for the general supersymmetric Lagrangian (11) $+(16)$.

Let us now analyse the $N=2$ supersymmetry transformations leaving invariant the three dimensional Born-Infeld SUSY theory. We shall not write the complete set of transformations but just those which are relevant for the discussion of Bogomol'nyi equations, namely those for the higgsino and gaugino (which we call $\psi$ and $\Sigma$ ):

$$
\begin{gathered}
\delta_{\epsilon} \psi=-i \not D \phi \epsilon=\left(\begin{array}{cc}
0 & D_{1}+i D_{2} \\
D_{1}-i D_{2} & 0
\end{array}\right)\left(\begin{array}{c}
\epsilon_{+} \\
\epsilon_{-}
\end{array}\right) \\
\delta_{\epsilon} \Sigma=\left(\frac{1}{2} \varepsilon_{\mu \nu \alpha} F^{\mu \nu} \gamma^{\alpha}+D\right) \epsilon=\left(\begin{array}{cc}
\frac{1}{2} \varepsilon_{i j} F^{i j}+D & 0 \\
0 & \frac{1}{2} \varepsilon_{i j} F^{i j}-D
\end{array}\right)\left(\begin{array}{l}
\epsilon_{+} \\
\epsilon_{-}
\end{array}\right)
\end{gathered}
$$

where we call $\epsilon$ the Dirac fermion transformation parameter (we have already made $N=A_{0}=0$ and considered the static case).

As it is well-known, making zero half of the SUSY variations associated with the higgsino and gaugino fields, one gets the Bogomol'nyi equations. For instance, by demanding that those generated by $\varepsilon_{+}$be zero, one gets the following self-dual equation from the higgsino's variation

$$
\delta_{\epsilon_{+}} \psi=0 \rightarrow D_{1} \phi=i D_{2} \phi
$$

One should note that this transformation law just depends on the way the parallel displacement is defined in terms of the gauge connection and not on the explicit form of the gauge field action. One can then understand why eq.(26) is completely independent 
of the particular form the gauge field action is chosen, at least for minimally coupled gauge theories Đ. Regarding the equation derived from the gaugino transformation,

$$
\delta_{\epsilon_{+}} \Sigma=\frac{1}{2} \varepsilon_{i j} F^{i j}+D=0
$$

it could, in principle, depend on the particular Lagrangian chosen through the $D$ term. However, as we have seen (eq.(22) ), the solution to the equation of motion for $D$ takes the same simple form for any gauge field Lagrangian since $D$ always enters through the combination $D^{2}-B^{2}$.

This feature can be also checked by analyzing the two supercharges which can be obtained following the usual Noether construction. As it has been shown in [14 for the Born-Infeld case, supercharges $Q$ and $\bar{Q}$ can be always put in the form

$$
\begin{aligned}
\bar{Q} & =i \int d^{2} x \Sigma^{\dagger} \mathcal{H}[B, D]\left(\gamma^{0} B+D\right)+\frac{i}{2} \int d^{2} x \psi^{\dagger} \not D \phi \\
Q & =-i \int d^{2} x\left(B+\gamma^{0} D\right) \mathcal{H}[B, D] \Sigma-\frac{i}{2} \int d^{2} x \gamma^{0}(\not D \phi)^{\dagger} \psi
\end{aligned}
$$

with $\mathcal{H}$ some real functional of $D$ and $B$ which can be computed order by order in $1 / \beta^{2}$. Furthermore, eqs. (28) also hold when one considers not just SUSY Born-Infeld theory but the general Lagrangian, viz. eq.(11). Only the actual form of $\mathcal{H}$ will change, depending on the different sets of possible $a^{\prime} s$ coefficients. What one can easily see is that the following formula holds

$$
\mathcal{H}=\mathcal{H}_{\text {Maxwell }}+\sum_{n=1}^{\infty} \frac{1}{\beta^{2 n}} \mathcal{H}_{n}[B, D]
$$

with

$$
\begin{gathered}
\mathcal{H}_{\text {Maxwell }}=1 \\
\left.\mathcal{H}_{n}[B, D]\right|_{B^{2}=D^{2}}=0
\end{gathered}
$$

It is clear that condition $\bar{Q}|p h y s\rangle=0$ is satisfied whenever $\left(B+\gamma^{0} D\right) \epsilon=0$ and $\not D \phi \epsilon=0$, independently of the precise form the functional $\mathcal{H}$ takes. Choosing just the upper component of the transformation parameter, $\epsilon_{+}$, yields again the two Bogomol'nyi equations $(23),(26)$. Of course, this is to be expected since both $\left(B+\gamma^{0} D\right) \epsilon$ and $\not D \phi \epsilon$, appearing in (28), provide the transformation laws of gaugino and higgsino respectively.

\footnotetext{
${ }^{1}$ For an analysis of Bogomoln'yi equations in non-minimally coupled gauge theories, see ref. 21 .
} 
Concerning the supercharge algebra, when the Bogomol'nyi equation $B= \pm D$ is used, only the Maxwell part of $\mathcal{H}$ survives, this showing again why the BPS structure is not sensitive to the particular form of the gauge field Lagrangian.

In conclusion, we have analysed the most general Lagrangian corresponding to the supersymmetric extension of a general bosonic Lagrangian depending on the two algebraic Maxwell invariants $F^{\mu \nu} F_{\mu \nu}$ and $\tilde{F}^{\mu \nu} F_{\mu \nu}$. This general Lagrangian includes, for a particular choice of coefficients, the Born-Infeld supersymmetric Lagrangian, and also an infinite class of Lagrangians having causal propagation [9]. We have shown why the Bogomol'nyi relations associated with the bosonic sector remain unchanged in spite of of the actual form of the gauge field Lagrangian: Maxwell, Born-Infeld or more complicated non-polynomial Lagrangians all have the same BPS structure.

Finally, we note that a similar analysis could be in principle undertaken for the case of non-abelian gauge theories. There is in this respect some ambiguity about the way one should define the trace structure of the action. For the Born-Infeld case, it is pointed out in [22] that the symmetrised trace defined in [23] seems to be singled out by BPS considerations with respect to other definitions, this suggesting the existence of a supersymmetric extension of such an action but not in those with other trace structure. We think precisely that a combined analysis of the general supersymmetry transformations and the equation of motion for the auxiliary field analogous to that presented here will show what kind of actions admit BPS relations. We hope to report on this issue in a separate work.

Acknowledgements: H.R.C. is partially supported by FAPERJ, Rio de Janeiro, Brazil, and C.N. by CONICET, Argentina. F.A.S. is partially supported by CICBA, CONICET, Fundación Antorchas, Argentina and a Commission of the European Communities contract No:C11*_CT93-0315.

\section{References}

[1] E.S. Fradkin and A.A. Tseytlin, Phys. Lett. 163B (1985) 123; A.A. Tseytlin, Nucl. Phys. B276 (1986) 391.

[2] R.G. Leigh, Mod. Phys. Lett. A4 (1989) 2767 and 2073.

[3] J.Polchinski, Tasi Lectures on D-Branes, hep-th/9611050. 
[4] M. Aganagic, C. Popescu and J.H. Schwarz, Phys. Lett. B393 (1997) 311; Nucl. Phys. B 495 (1997) 99.

[5] J. Bagger and A. Galperin, Phys. Rev. D 55 (1997) 1091.

[6] C.G. Callan and J.M. Maldacena, Nucl. Phys. B 513 (1998) 198.

[7] G. Gibbons, Nucl. Phys. B 514 (1998) 603.

[8] J.P. Gauntlett, J. Gomis and P.K. Townsend, JHEP 01(1998) 003, hep-th 9711205 .

[9] S. Deser and R. Puzalowski, J. Phys. A13 (1980) 2501.

[10] S. Ceccotti and S. Ferrara, Phys. Lett. B187 (1987) 335.

[11] T. Hagiwara, Nucl. Phys. B 189 (1981) 135

[12] K. Shiraishi and S. Hirenzaki, Int. Jour. of Mod. Phys. A6 (1991) 2635.

[13] A. Nakamura and K. Shiraishi, Hadronic Jour. 14 (1991) 369.

[14] S. Gonorazky, C. Núñez, F.A. Schaposnik and G. Silva, Bogomol'nyi Bounds and the Supersymmetric Born-Infeld Theory, hep-th/9805054, Nucl. Phys. B in press.

[15] M. Born, Proc. R. Soc. London A143 (1934) 410.

[16] M. Born and M. Infeld, Proc. R. Soc. London A144 (1934) 425.

[17] E.B. Bogomol'nyi, Sov. Jour. Nucl. Phys. 24 (1976) 449. (1987) 335.

[18] H. de Vega and F.A. Schaposnik, Phys. Rev D (1976) 1100.

[19] H. B. Nielsen and P. Olesen, Nucl. Phys. B61 (1973) 45.

[20] J.D. Edelstein, C. Núñez, F.A. Schaposnik, Phys. Lett. B329 (1994) 39.

[21] H.R. Christiansen, M.S. Cunha, J.A. Helayël-Neto, L.R.U. Manssur and A.L.M. Nogueira Self-dual vortices in a Maxwell Chern-Simons model with non-minimal coupling, hep-th/9805128.

[22] D. Brecher, BPS states of the non-Abelian Born-Infeld action, hep-th/9804180

[23] A.A. Tseytlin, Nucl. Phys. B501 (1997) 41. 